\title{
The correlation between colorectal cancer rates of proliferation and apoptosis and systemic cytokine levels; plus their influence
} upon survival

\author{
C Evans', I Morrison', AG Heriot', JB Bartlett', C Finlayson', AG Dalgleish' and D Kumar*,I \\ 'Colorectal Surgery Unit \& Division of Oncology, St James Wing (Level III), St George's Hospital, Blackshaw Road, London SWI 7 OQT, UK
}

\begin{abstract}
Colorectal cancer development is associated with a shift in host immunity with suppression of the cell-mediated immune system $(\mathrm{CMI})$ and a predominance of humoral immunity $(\mathrm{HI})$. Tumour progression is also associated with increased rates of cell proliferation and apoptosis. The aim of this study was to investigate whether these factors correlate and have an influence upon prognosis. Longterm follow-up was performed on 40 patients with colorectal cancer who had levels of tumour necrosis factor (TNF)- $\alpha$, interferon (IFN)- $\gamma$ and interleukin (IL)- 10 measured from stimulated blood cultures before surgery. Their archived tumour specimens were analysed to determine a Ki-67-derived proliferation index (PI) and a M30-derived apoptosis index (Al). Tumour necrosis factor- $\alpha$ levels negatively correlated to tumour proliferation $(\rho=-0.697, P=0.01)$. Interleukin- 10 levels had a positive correlation with tumour proliferation $(\rho=0.452, P=0.05)$ and apoptosis $(\rho=0.587, P=0.01)$. Patient survival correlates to tumour pathological stage $(P=0.0038)$ and vascular invasion $(P=0.00 \mid 4)$. An $A l \leqslant 0.6 \%$ and TNF- $\alpha$ levels $\geqslant 8 \mid 48 \mathrm{pg} \mathrm{ml}^{-1}$ correlate to improved survival $(P=0.032, P=0.021)$. Tumour proliferation and apoptosis correlate to progressive suppression of the CMI-associated cytokine TNF$\alpha$ and to and higher levels of IL-10. Survival is dependent upon the histological stage of the tumour, vascular invasion, rates of apoptosis and proliferation and systemic immunity which are all interconnected.
\end{abstract}

British Journal of Cancer (2006) 94, 1412-1419. doi:10.1038/sj.bjc.6603104 www.bjcancer.com

Published online 25 April 2006

(C) 2006 Cancer Research UK

Keywords: colorectal cancer; cytokines; immune suppression; proliferation: apoptosis; survival

The basis for the development of colorectal cancer is believed to be the adenoma-carcinoma sequence (Weitz et al, 2005), which involves a stepwise progression from normal to dysplastic epithelium to carcinoma. It is associated with the accumulation of multiple, clonally selected genetic alterations (Leslie et al, 2002) and results in disturbed rates of cell proliferation and apoptosis (Aotake et al, 1999).

Cell proliferation and apoptosis are dependent upon the pathways that govern the different cell cycle phases. These pathways are under genetic control but are influenced by both exogenous and endogenous stimuli (Funk, 1999). The immune system and its components are key regulators of cell proliferation and cell death when there are inflammatory responses, for example, during wound healing (Robinson and Coussens, 2005). The majority of colorectal cancer evolves in a chronic inflammatory background (Higaki et al, 1999) and there is strong evidence of an immune response to colorectal cancer (Naito et al, 1998; Garrido and Algarra, 2001; Nagorsen et al, 2005).

In order for tumours to establish and progress it is thought that suppression of the host's immune system is needed (Berghella et al, 1996). Tumour-induced immune suppression occurs at both

*Correspondence: Professor D Kumar; E-mail: dkumar@sgul.ac.uk Received 12 January 2006; revised 20 February 2006; accepted 9 March 2006; published online 25 April 2006 a molecular and cellular level and progresses to affect the whole organism (Zou, 2005). Colorectal cancer results in a fundamental shift in immunity with suppression of the cell-mediated immune system (CMI), associated with tumour rejection (Kidd, 2003), and a predominance of humoral immunity (HI) which conversely prevents rejection (Terabe et al, 2004). There is a reduced production of cytokines from the CMI-associated Th1 lymphocytes while those produced by the Th2 lymphocytes (associated with HI) appear to remain at normal or even elevated levels. The further the disease has progressed the more significant these imbalances become and complete resection of the tumour reverses these changes (O'Hara et al, 1998; Heriot et al, 2000; Galizia et al, 2002; Shibata et al, 2002).

The purpose of this study was to correlate colorectal tumour rates of proliferation and apoptosis with host systemic cytokine levels and investigate what influence these factors have upon patient prognosis. Archived cancer specimens from a group of patients, who were previously studied to investigate the relationship between systemic cytokine levels and colorectal cancer (Heriot et al, 2000), were stained with the antibody Ki67 to measure proliferation and the antibody M30 to measure apoptosis. Long-term patient follow-up was also preformed. The original work had shown a suppression of CMI-associated cytokines, tumour necrosis factor- $\alpha$ (TNF- $\alpha$ ) and interferon- $\gamma$ (IFN- $\gamma$ ) in patients with colorectal cancer that was greater in those with a Dukes' C tumour compared to those with either a Dukes' A or B tumour and was reversed by surgery. No difference had been 
found in the levels of the HI-associated cytokine interleukin-10 (IL-10) in patients with colorectal tumours compared to the controls both pre- and postsurgery (Heriot et al, 2000).

\section{PATIENTS AND METHODS}

Between March 1997 and March 1998, 53 patients (34 male patients) diagnosed at one institution with primary colorectal cancer were recruited. All patients gave written, informed consent to the study. Ethical approval for the study was obtained from the local ethical committee.

All patients had adenocarcinoma of the colon and rectum. Before treatment a single heparinised venous blood sample was taken from each patient for analysis. Following surgery a further venous samples was taken a minimum of 4 weeks postsurgery to be used for postoperative data analysis. The blood was diluted with RPMI-1640 medium (1:4) + glutamine (2 mM) and then stimulated for $24 \mathrm{~h}$ with lipopolysaccharide. Cell-free supernatants were then assayed for TNF- $\alpha$, IFN- $\gamma$ and IL-10 by enzyme-linked immunosorbent assay (ELISA) using an assay procedure and reagents (anticytokine capture monoclonal antibody, biotinylated anticytokine detecting antibody and recombinant cytokine) provided by Pharmingen (Cambridge Bioscience, UK) (Heriot et al, 2000).

Clinical follow-up was performed every 3 months for the first 2 years after treatment, 6 monthly during the third year and annually thereafter till the age of 80 years. In December 2004 patients with preoperative cytokine levels were followed up and their archived pathological specimens were reviewed retrospectively. Patient's who had chemotherapy and/or radiotherapy before surgery, patients with synchronous, or metachronous tumours, inflammatory conditions or any immunosuppressive condition including immunosuppressive medications were excluded. The interval from the date of resection to the date of death or last contact (if alive) was used for survival analysis with a minimum length of follow-up set at 5 years. The end points of the follow-up study were; cancerrelated survival and the development of local recurrence or distant metastases.

\section{Immunohistochemistry}

Sections (3 im thick) were cut from paraffin blocks on to SuperFrost ${ }^{\circledR}$ Plus slides (Menzel-Glaser, Germany), dried overnight at $60^{\circ} \mathrm{C}$, dewaxed and taken down to water. Endogenous peroxidase activity was blocked by placing slides in $500 \mathrm{ml}$ of a $10 \%$ hydrogen peroxidase solution for $8 \mathrm{~min}$. Slides were washed in tap water and heated for $8 \mathrm{~min}$ in $1000 \mathrm{ml}$ of Tris-EDTA-citrate buffer, pH 7.8 (preheated for $24 \mathrm{~min}$ ) in a microwaveable pressure cooker if for Ki67 staining and for $35 \mathrm{~min}$ in $1000 \mathrm{ml}$ of Tris-EDTA pH 9.9 in an unpressurised container if for M30 staining. After repeat washing, the slides were ringed with a hydrophobic ImmEdge $^{\mathrm{TM}}$ pen (Vector Laboratories Inc., Bulingame, CA 94010, USA) and rinsed in Optimax Wash Buffer ${ }^{\mathbb{B}}$ (Biogenex Laboratories, San Ramon, CA, USA). The primary antibodies NCL-Ki67p (Novocastra Laboratories Ltd, Newcastle upon Tyne, England, UK) and M30 CytoDEATH (Roche Applied Science, Lewes, England, UK) were applied to the sections according to the Optimax stainer programme having been diluted to $1: 6000$ and $1: 50$, respectively, with ChemMate ${ }^{\mathrm{TM}}$ Antibody Diluent (DakoCytomation). The stainer was programmed using Optimax ${ }^{\mathrm{TM}}$ Software Version 2.7 to the following specifications: (1) incubation in primary antibody, 30 and $45 \mathrm{~min}$, respectively, (2) 30 or $45 \mathrm{~min}$, respectively, in polymetric conjugate, (3) $10 \mathrm{~min}$ in working substrate solution; rinsing between all stages with Optimax wash buffer ${ }^{\circledR}$. Slides stained with M30 had the working substrate solution (ChemMate ${ }^{\mathrm{TM}}$ Envision $^{\text {TM }}$ Detection kit, Peroxidase/DAB, Rabbit/mouse (DakoCytomation) applied $45 \mathrm{~min}$ after the polymetric conjugate. On completion of the staining, the slides were washed in tap water, and counterstained with Mayer's haematoxylin for $1 \mathrm{~min}$ and then differentiated briefly in $1 \%$ acid alcohol, blued in running tap water, dehydrated cleared and mounted.

\section{Evaluation of staining results}

All specimens were examined by an independent investigator with no knowledge of the clinical or pathological data. Counts were made randomly from each slide with at least 1000 epithelial cells counted in all cases. Ki67 positivity and M30 positivity were identified and positive cells were expressed as a percentage of the total number of cells counted (proliferation index (PI) and apoptotic index (AI)) (Figures 1 and 2).

\section{Statistical analysis}

Results are noted as mean $+/-$ standard deviation (s.d.). Correlations between proliferation and apoptotic activity and systemic cytokine levels were assessed using the Spearman test. Independent $t$-tests were used to analyse means. In order to perform survival analysis and create probability curves dependent upon tumour rates of proliferation and apoptosis as well as systemic cytokine levels the Kaplan-Meier method was used. A log-rank test was used to compare the probability curves. Hazard ratios and $95 \%$ confidence intervals (CI) for cytokine values, PI, AI, Dukes' stage, differentiation and vascular invasion were calculated for each factor in a Cox proportional hazards model. In all cases, a $P$-value of $<0.05$ indicated a significant difference. All data were analysed using SPSS ${ }^{\circledR}$ Version 12.0 (SPSS Inc., Chicago, IL, USA).

\section{RESULTS}

\section{Patient characteristics}

From the original 53 patients, 40 (26 male patients) were eligible for the study. Of them, 11 were excluded for having no

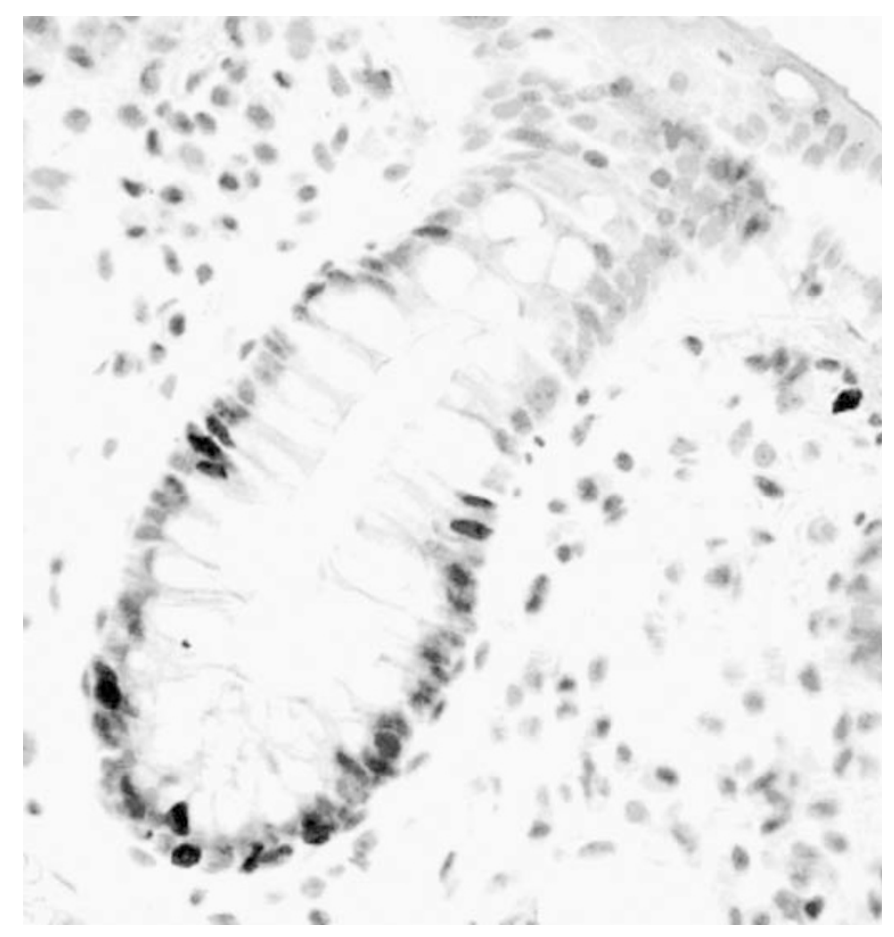

Figure I Histological section stained with anit-Ki67, I:6000. Pretreatment Tris-EDTA pH7.6 (magnification $\times 200$ ). 


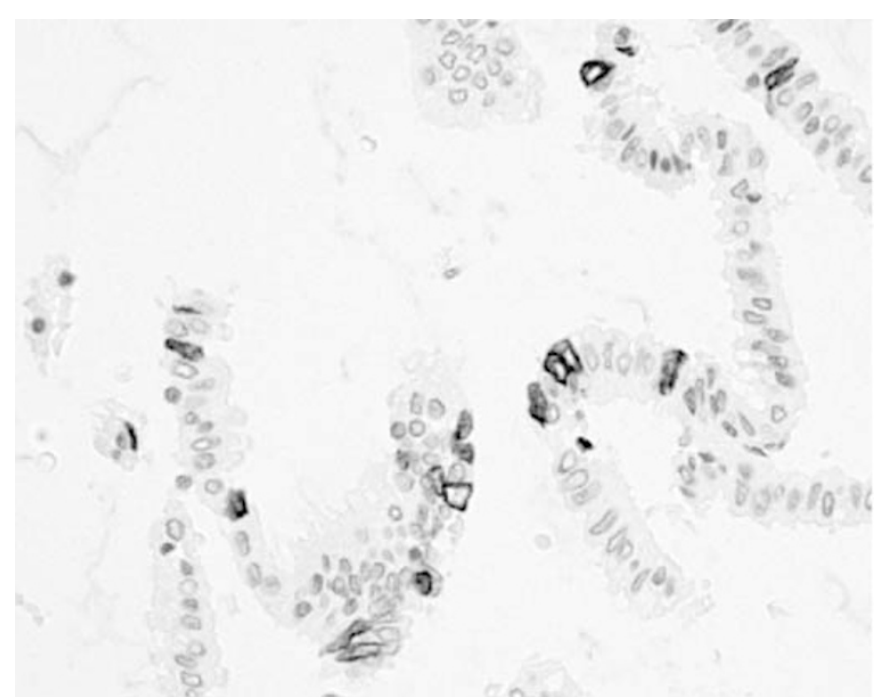

Figure 2 Histological section of colon stained with M30CytoDEATH I: 50 TE buffer in the Decloaker (magnification $\times 200$ ).

preoperative cytokine values successfully measured, one for the tumour being a local recurrence and one for being lost to followup. Median age was 76.5 years old with a mean (s.d.) of 74.55 (11.80). In all, 35 were Caucasian, four were Asian and one was Afro Caribbean. From this group of 40 patients, it was possible to retrieve and successfully stain 31 histological specimens for proliferation and apoptosis.

In December 2004, the median follow-up was 89 months (mean (s.d.) 87.64 (4.02) months). Of the patients, four had Dukes' A tumours, 16 Dukes' B tumours and the remaining 20 had Dukes' $\mathrm{C}$ tumours. In all, 24 patients were documented to have well or moderately differentiated tumours while 15 had poorly differentiated tumours. Vascular invasion was found in 14 patient's specimens. Overall mortality was $65 \%$ (26 patients) with a cancerrelated mortality of $50 \%$ (20 patients). Median survival was 21.5 months with a mean (s.d.) of 35.88 (33.64) months. Six patients at the time of surgery had metastases with an additional five developing metastases during follow-up. Five patients developed local recurrence postsurgery. Cancer-related 5-year mortality in this cohort of patients is noted to be high but was comparable to national statistics (Cancer Stats: Large Bowel-UK, Cancer Research UK, 2005) given the small number of patients involved.

\section{Proliferative and apoptosis activity}

The mean (s.d.) PI in the colorectal carcinomas was 52.39 (19.50) $\%$. The mean (s.d.) AI was 0.74 (0.6) \%. Proliferative index and AI did not correlate to Dukes' histological stage but, as a combined group, those patients who developed either local recurrence or metastases had a significantly raised PI $(P=0.002)$ and AI $(P=0.037)$. There was a strong positive correlation between AI and PI $(\rho=0.675, P=0.01)$.

\section{Correlation between systemic cytokine levels and tumour rates of proliferation and apoptosis}

A significant negative correlation was found between systemic TNF- $\alpha$ levels and tumour cell proliferation $(\rho=-0.697, P=0.01)$ (Figure 3). A trend to negative correlation was seen when TNF- $\alpha$ levels were correlated to apoptosis $(\rho=-0.33)$ and IFN $-\gamma$ levels to proliferation $(\rho=-0.265)$. Patient systemic IL-10 levels had a positive correlation with both tumour cell proliferation $(\rho=0.452$, $P=0.05)$ and apoptosis $(\rho=0.587, P=0.01)$ (Figure 4(i) and (ii)).

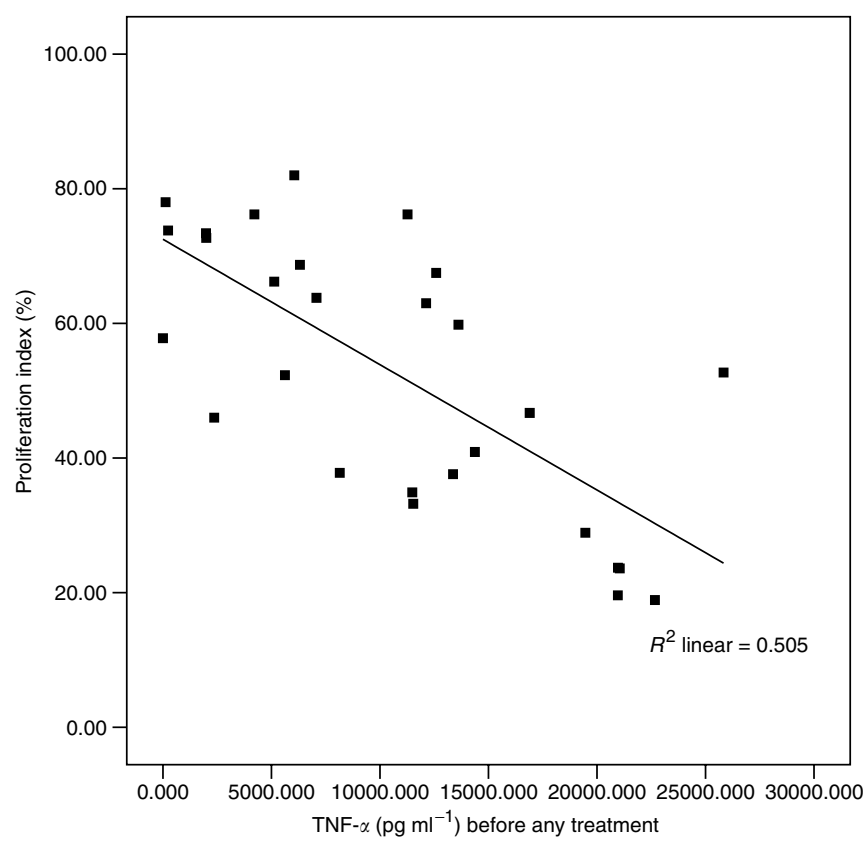

$\rho=-0.697, P=0.01$

Figure 3 Correlation between preoperative TNF- $\alpha$ levels $\left(\mathrm{pg} \mathrm{ml}^{-1}\right)$ and tumour PI.

\section{Correlations to long-term prognosis}

It was shown that the tumour's pathological staging was of significance when assessing which factors influenced survival. In keeping with the expected survival distribution of patients with colorectal cancer, those with Dukes' $C$ tumours had a poorer survival compared to those with either a Dukes' A or B tumour $(P=0.0038)$. Vascular invasion was also correlated to reduced survival $(P=0.0014)$ (Figure 5(i) and (ii)).

Tumour rates of proliferation and apoptosis Patients who had an AI less than or equal to the median value of $0.6 \%(n=16)$ had significantly better overall survival than those whose value was greater $(n=15)$ (log-rank $P=0.032)$ (Figure 6(i)). Patients who had a PI less than or equal to the median value of $52.7 \%(n=16)$ showed a strong trend to improved survival (log-rank $P=0.051$ ) (Figure 6(ii))

Systemic cytokine levels Using independent $t$-tests preoperative cytokine levels were compared to see what correlation they had with long-term survival, development of metastases and local recurrence. Patients with a survival of greater than 1 year had significantly higher levels of preoperative TNF- $\alpha$ than those that did not $(P=0.005)$. There was no correlation between preoperative IFN- $\gamma$ and IL-10 levels and 1-year survival. Preoperative cytokine levels were not significantly different in those who developed metastases or local recurrence compared to those who did not and there were no statistically significant correlations with postoperative cytokine levels.

In order to perform survival analysis patients were classified in to two groups according to their cytokine values. When assessing the influence of TNF- $\alpha$ patients were grouped as either having values $\leqslant$ the median value of $8148 \mathrm{pg} \mathrm{ml}^{-1} \quad(n=17)$ or $>8148 \mathrm{pg} \mathrm{ml}^{-1} \quad(n=18)$. To assess IL-10 patients were split depending whether their values were $>$ or $\leqslant$ the median value of $0.4255 \mathrm{OD}_{450}(n=17$ and $n=16$, respectively). However, to assess IFN- $\gamma$ patients were split in to those with values detectable 

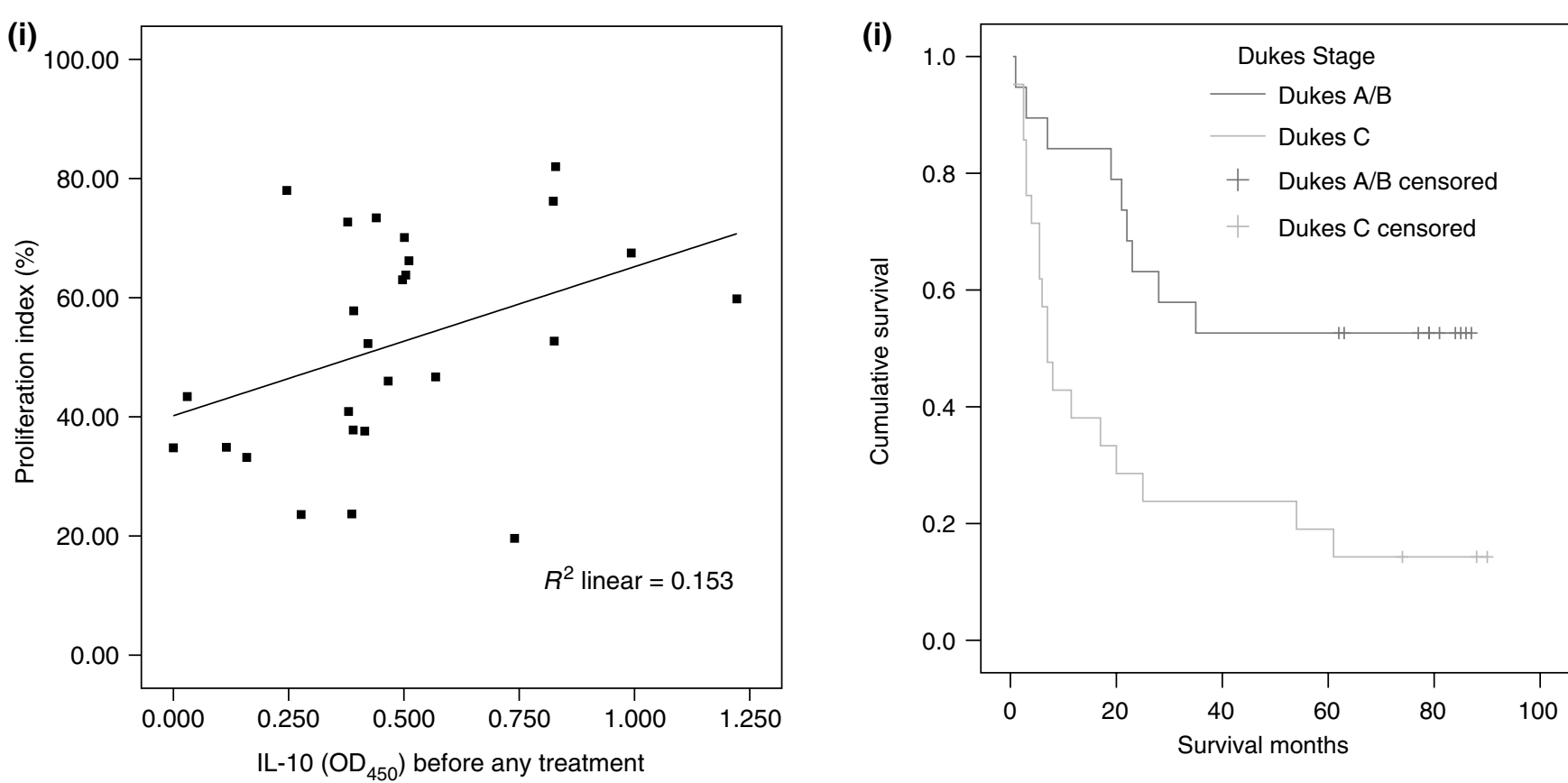

$\rho=0.452, P=0.05$

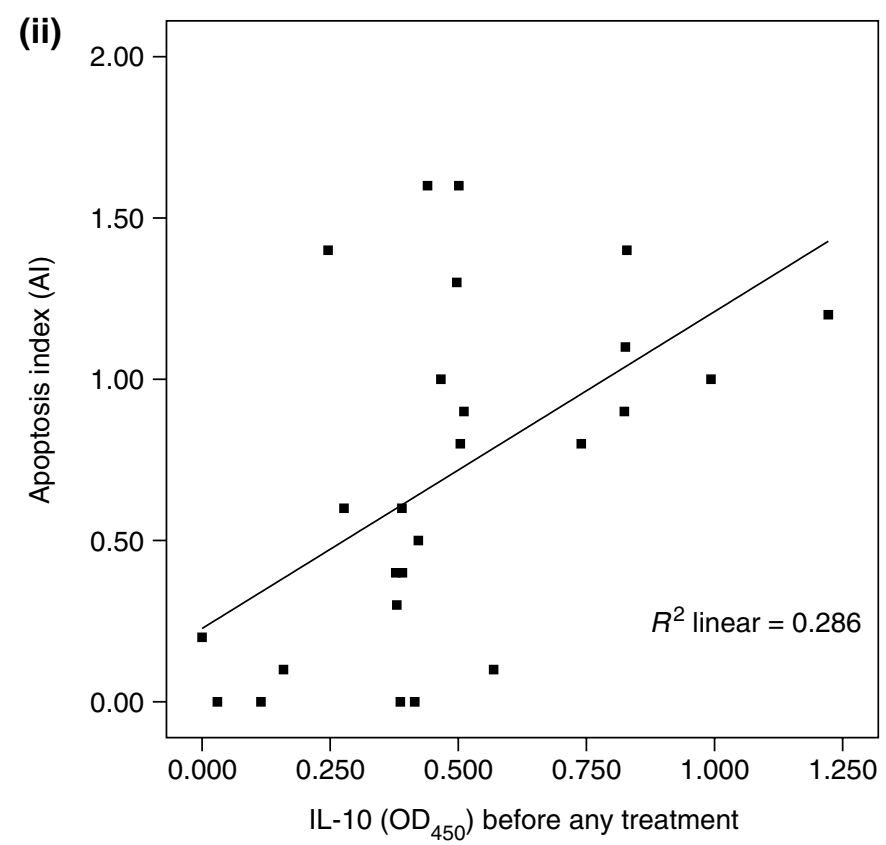

$\rho=0.587, P=0.01$

Figure 4 (i) Correlation between preoperative IL- 10 levels $\left(O D_{450}\right)$ and tumour PI. (ii) Correlation between preoperative IL- IO levels $\left(\mathrm{OD}_{450}\right)$ and tumour Al.

$\left(>4.97 \mathrm{pg} \mathrm{ml}^{-1}\right)(n=12)$ or not detectable $\left(\leqslant 4.7 \mathrm{pg} \mathrm{ml}^{-1}\right)(n=26)$ owing to a skewed patient distribution. Patients with TNF- $\alpha$ levels $>8148 \mathrm{pg} \mathrm{ml}^{-1}$ had significantly better survival than those with TNF- $\alpha \leqslant 8148 \mathrm{pg} \mathrm{ml}^{-1}(P=0.021)$ (Figure $\left.7(\mathrm{i})\right)$. Patients with detectable IFN- $\gamma$ levels showed a trend to improved survival compared to those with undetectable levels, however, not reaching significance $(P=0.148)$ (Figure $7(\mathrm{ii}))$. There was no statistical difference between the two IL-10 groups although at 60 months it is noted that cumulative survival is more than double in the group with values $\leqslant 0.4255 \mathrm{OD}_{450}(0.41$ vs 0.18$)$ (Figure $7(\mathrm{iii})$ ). 


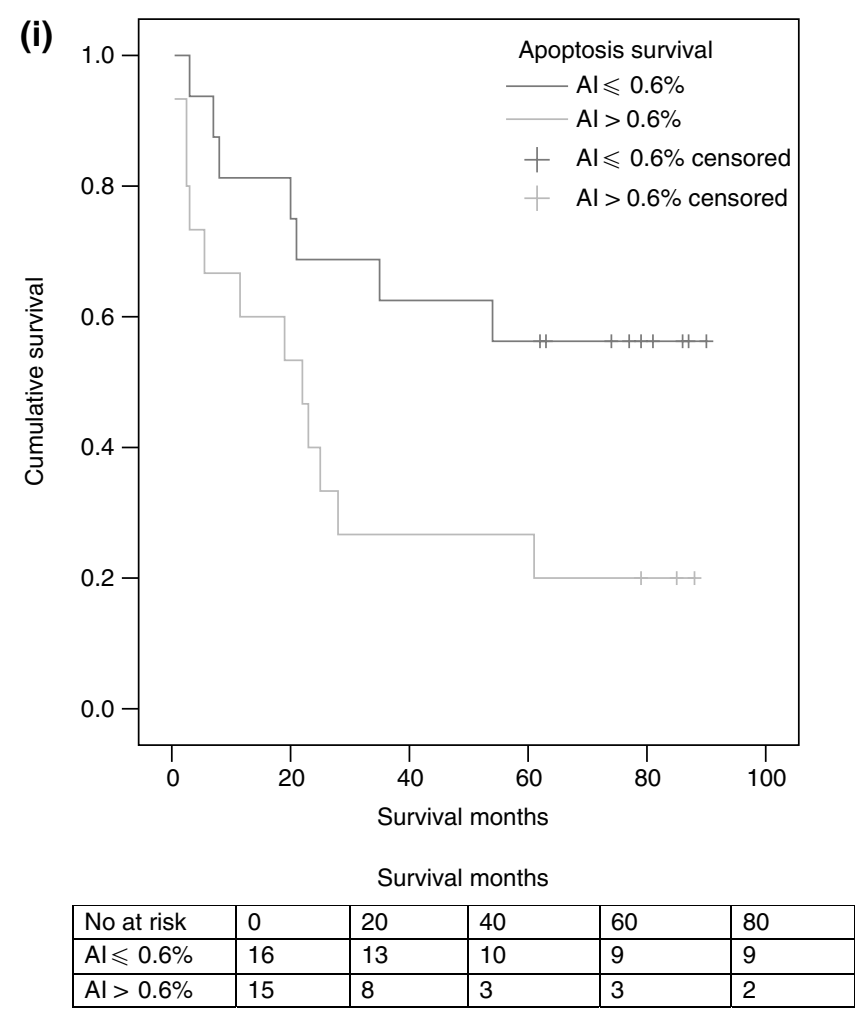

$P=0.032$

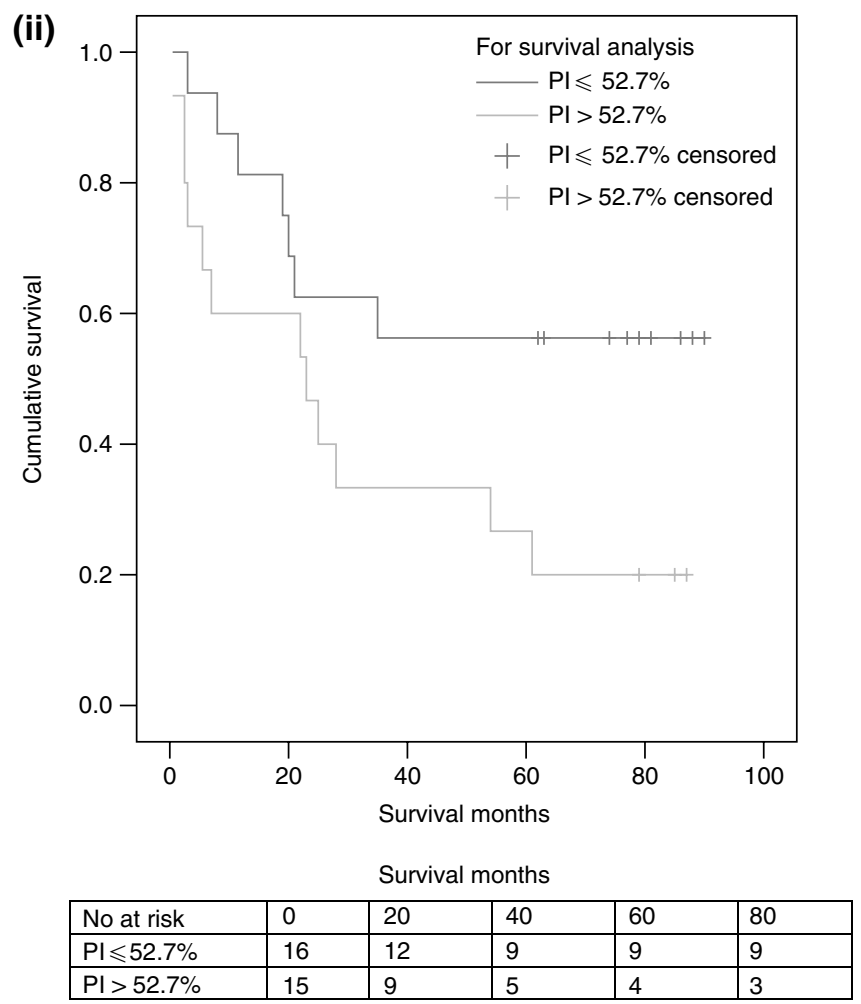

$P=0.051$

Figure 6 (i) Al survival functions. (ii) Pl survival functions.

\section{DISCUSSION}

Colorectal carcinomas arise through a series of histological changes, each of which is accompanied by specific genetic alterations in a handful of oncogenes and tumour-suppressor genes (Fodde et al, 2001). Chronic inflammation plays a causative role in the molecular pathways of colon carcinogenesis (Itzkowitz and Yio, 2004) and leads to altered rates of cell proliferation and apoptosis, altered cellular adhesion, increased angiogenesis and cellular transformation (Macarthur et al, 2004). Significantly, chronic inflammation also causes negative feedback inhibition of the CMI and causes a predominance of HI. This state of host immunity is described in a number of malignant diseases and inhibits immune-mediated tumour rejection (O'Byrne and Dalgleish, 2001).

Colorectal cancer has been shown to cause CMI suppression and $\mathrm{HI}$ promotion that is linked to disease progression and has an influence on survival. Decreased total numbers of the CMI promoting Th1 $\mathrm{CD} 4{ }^{+}$cells have been found in patients with colorectal tumours (Nakayama et al, 2000). Lowered levels of CMIassociated cytokines have been measured, corresponding to the pathological stage of the tumour and reversed by surgery (O'Hara et al, 1998; Heriot et al, 2000; Galizia et al, 2002; Shibata et al, 2002). It has also been demonstrated that elevated basal levels of the HI-associated cytokine, IL-10, are correlated to reduced disease-free survival (Galizia et al, 2002). The mechanisms through which this shift in immunity is performed have not been fully explained but include COX-2 overexpression (O'Byrne and Dalgleish, 2001), histamine production (Tomita and Okabe, 2005) and increased expression of IL-10 (Ding et al, 2003).

There is evidence of an immune response to colorectal cancer which can be correlated to improved survival (Naito et al, 1998); however, many of the cell types active in chronic inflammation can be found in the surrounding stroma and the neoplasm itself. This includes a diverse leucocyte infiltrate of macrophages, neutrophils, eosinophils and mast cells (Macarthur et al, 2004). Thus, the secretion of extracellular proteases, proangiogenic factors, and cytokines in the tumour microenvironment results in a chronic inflammatory-like state in which CMI suppression takes place and the control mechanisms for cell proliferation and apoptosis are disturbed.

Despite there being only a small number of patients involved in this study, it is the consistency in its results, relating rates of proliferation and apoptosis to systemic cytokine levels and the trends seen when looking at long-term survival, that suggest progressive changes to host immunity with colorectal cancer advancement. Tumour rates of proliferation and apoptosis have a negative correlation with the CMI-associated cytokines TNF- $\alpha$ and IFN- $\gamma$ while having a positive one with IL-10 (HI associated). Elevated AI and PI were linked with reduced long-term survival and the development of metastases and/or local recurrence. Correspondingly, reduced rates of survival are seen with lowered levels of TNF- $\alpha$. It is not possible to comment from these data whether a specific cytokine level or tumour proliferation or apoptosis rate has a prognostic value and it should be noted that a larger range of cytokines and T-cell responses would need to be measured to make a full evaluation of any shift in host immunity from CMI to HI. However, it can be concluded that there is an impact upon patient survival through the systemic suppression of the CMI-associated cytokines TNF- $\alpha$ and IFN- $\gamma$ and promotion of the HI-associated IL-10.

The balance between cell proliferation and cell loss through apoptosis determines how fast a tumour grows and is an important determinant of tumour behaviour (Koornstra et al, 2003). There are conflicting reports regarding the implications of proliferation and apoptosis rates upon patient prognosis (Koornstra et al, 2003; Salminen et al, 2005; Valera et al, 2005), however, it is generally accepted that as tumours advance proliferative activity increases and higher rates of apoptosis are seen (Koornstra et al, 2004). This study's results conform to these findings demonstrating increased rates of proliferation and apoptosis in the group of patients developing either metastatic disease or local recurrence with a 


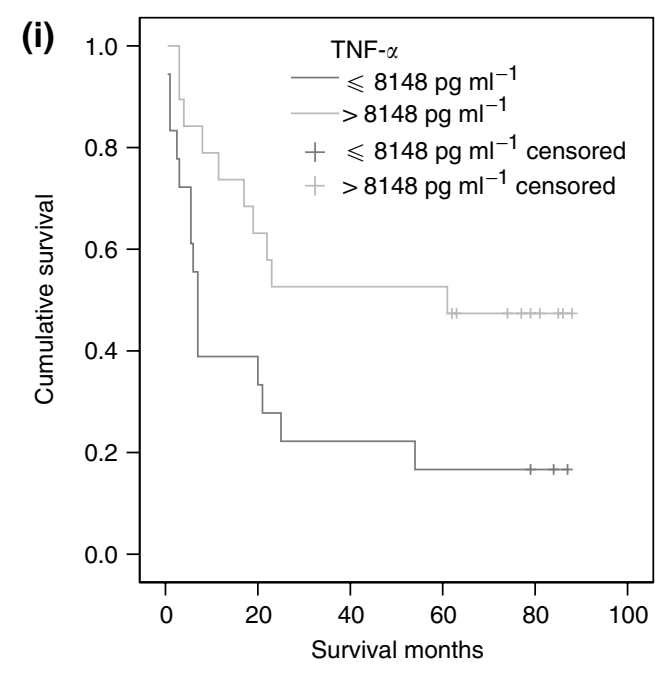

Survival months

\begin{tabular}{|l|r|r|r|r|r|}
\hline No at risk & 0 & 20 & 40 & 60 & 80 \\
\hline TNF- $\alpha>8,148 \mathrm{pg} \mathrm{ml}^{-1}$ & 18 & 11 & 9 & 8 & 7 \\
\hline TNF- $\alpha \leqslant 8,148 \mathrm{pg} \mathrm{ml}^{-1}$ & 17 & 6 & 3 & 2 & 2 \\
\hline
\end{tabular}

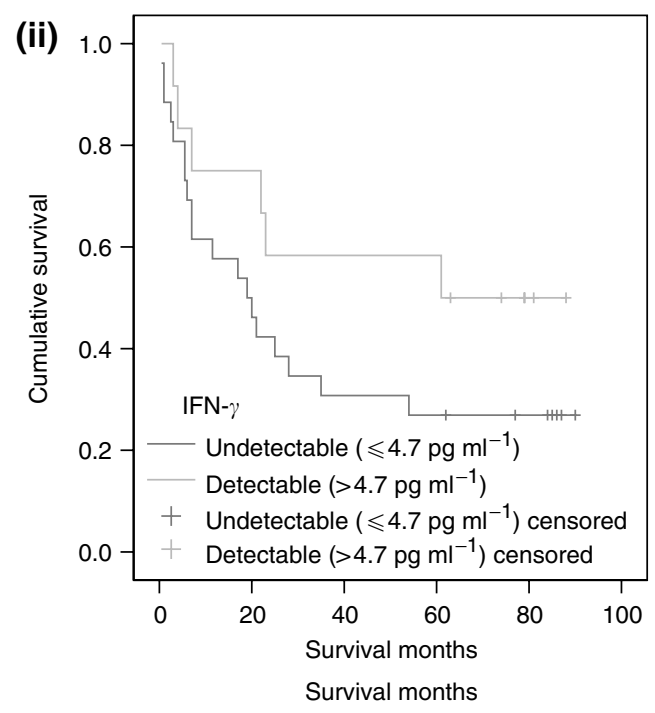

\begin{tabular}{|l|r|r|r|r|r|}
\hline No at risk & 0 & 20 & 40 & 60 & 80 \\
\hline IFN- $\gamma$ detectabel $\left(>4.97 \mathrm{pg} \mathrm{ml}^{-1}\right)$ & 12 & 9 & 7 & 7 & 6 \\
\hline IFN- $\gamma$ undetectable $\left(\leqslant 4.97 \mathrm{pg} \mathrm{ml}^{-1}\right)$ & 26 & 12 & 8 & 7 & 7 \\
\hline
\end{tabular}

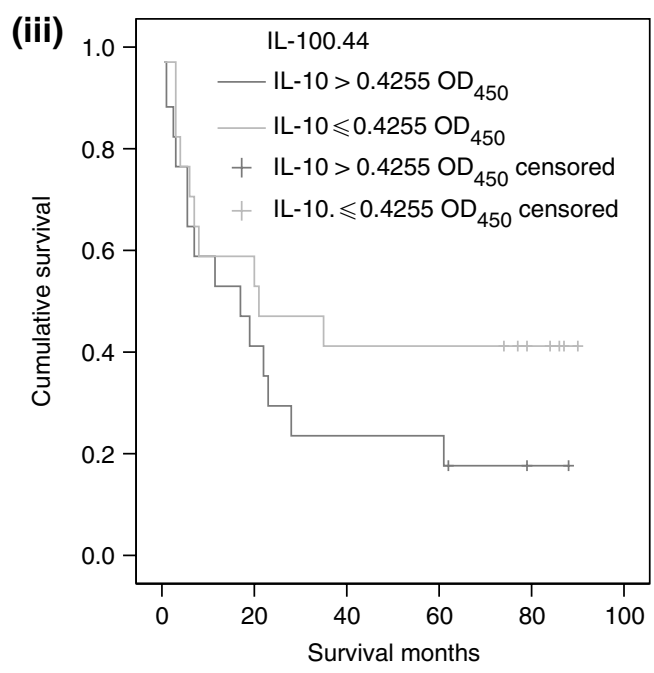

\begin{tabular}{|l|r|r|r|r|r|}
\hline So at risk & $\mathrm{O}$ & 20 & 40 & 60 & 80 \\
\hline $\mathrm{IL}-10>0.4255 \mathrm{OD}_{450}$ & 17 & 7 & 3 & 3 & 2 \\
\hline $\mathrm{IL}-10 \leqslant 0.4255 \mathrm{OD}_{450}$ & 16 & 9 & 7 & 6 & 6 \\
\hline$P=0.21$
\end{tabular}

Figure 7 (i) TNF- $\alpha$ survival functions. (ii) IFN- $\gamma$ survival functions. (ii) IL- I 0 survival functions.

Table I Multivariate analysis of significant factors associated with overall 5-year survival in colorectal cancer

\begin{tabular}{|c|c|c|c|c|}
\hline & Regression coefficient & s.e. & Hazard ratio & $P^{*}$ \\
\hline Differentiation (well/mod) vs (poor) & -2.065 & 0.88 & $0.127(0.23,0.71)$ & 0.019 \\
\hline Proliferation index (continuous variable) & -0.021 & 0.04 & $0.98(0.91,1.06)$ & 0.609 \\
\hline Apoptosis index (continuous index) & 6.691 & 3.05 & $805.12(2.02,320256.16)$ & 0.028 \\
\hline TNF- $\alpha\left(>8 / 48 \mathrm{pg} \mathrm{ml}^{-1}\right.$ vs $\left.\leqslant 8148 \mathrm{pg} \mathrm{ml}^{-1}\right)$ & -0.983 & 1.42 & $0.374(0.023,6.05)$ & 0.489 \\
\hline
\end{tabular}

\footnotetext{
Values in parentheses are $95 \% \mathrm{Cl}$. *Cox proportional hazards model.
} 
strong positive correlation between PI and AI also noted. The demonstrated link between increased rates of apoptosis and proliferation with reduced survival in this cohort of patients could possibly be explained by this pathological-proliferation/apoptosis correlation.

The relationship between cytokines and tumour development is complex with both stimulation and inhibition exhibited by the same one cytokine. Their action depends upon the cocktail of other factors in the surrounding microenvironment, including other cytokines, chemokines, growth and angiogenic factors as well as its own actual concentration. For example, TNF- $\alpha$ can induce apoptosis in tumour cells of the colon (Jacobson-Brown and Neuman, 2004) and its cytotoxic effect is synergistically enhanced by IFN- $\gamma$ in vitro and in vivo (Sveinbjornsson et al, 1997). However, when chronically produced TNF may act as an endogenous tumour promoter, contributing to tissue remodelling and stromal development necessary for tumour growth and spread (Balkwill and Mantovani, 2001). Interleukin-10 is believed to contribute to tumour development by suppressing the antitumour immune responses (Matsuda et al, 1994). Gene therapy studies, however, have demonstrated IL-10-mediated inhibition of tumour growth and metastases with the outcome appearing dependent upon host immune competency and levels of IL-10 expression (Erdman et al, 2003).

Measuring systemic levels of three cytokines cannot accurately represent the specific interactions occurring in the tumour microenvironment or give a full representation of systemic immunity. However, it does enable potential trends or changes in host immune competence to be proposed. Thus, this study suggests a potential shift in host immunity with CMI suppression and HI promotion, which correlates to increasing tumour rates of proliferation and apoptosis. The effect of any one single cytokine's is not implicated.

These results linking the host's immune response to colorectal cancer progression and prognosis highlight the need for immunotherapies, which enhance CMI. Manipulating the host's systemic immune response to a tumour may lead to an environment in which tumour cell recognition is more successfully induced (Shibata et al, 2002) and several studies have demonstrated that CMI-associated cytokines enhance the therapeutic efficacy of antitumour responses (Nakamori et al, 2003). Combining immunotherapies with other treatment modalities including chemotherapy and radiotherapy may also lead to more successful results.

\section{CONCLUSION}

There is a trend to TNF- $\alpha$ and IFN- $\gamma$ suppression with IL-10 promotion as colorectal cancer advances. Cytokine levels correlate to increased tumour rates of proliferation and apoptosis. Patient survival is influenced by the histological stage of the tumour, vascular invasion, tumour rates of apoptosis and proliferation and systemic immunity. All of these factors are interconnected. Immunotherapies that can enhance cell-mediated immunity may lead to prolonged survival and should be considered in combination with other treatments used to induce tumour recognition.

\section{REFERENCES}

Aotake T, Lu CD, Chiba Y, Muraoka R, Tanigawa N (1999) Changes of angiogenesis and tumor cell apoptosis during colorectal carcinogenesis. Clin Cancer Res 5: $135-142$

Balkwill F, Mantovani A (2001) Inflammation and cancer: back to Virchow? Lancet 357: $539-545$

Berghella AM, Pellegrini P, Del Beato T, Maccarone D, Adorno D, Casciani CU (1996) Prognostic significance of immunological evaluation in colorectal cancer. Cancer Biother Radiopharm 11: 355-361

Cancer Research UK (2005) Cancer stats - bowel (colorectal) cancer. www.cancerresearchuk.org

Ding YH, Fu S, Zamarin D, Bromberg J (2003) Interleukin-10. In The cytokine Handbook, 2 Volume Set, 4th Edition, Thompson AW, Lotze MT (eds) London, Academic Press, pp 603-625. Elsevier Science

Erdman SE, Rao VP, Poutahidis T, Ihrig MM, Ge Z, Feng Y, Tomczak M, Rogers AB, Horwitz BH, Fox JG (2003) CD4(+)CD25(+) regulatory lymphocytes require interleukin 10 to interrupt colon carcinogenesis in mice. Cancer Res 63: $6042-6050$

Fodde R, Smits R, Clevers H (2001) APC, signal transduction and genetic instability in colorectal cancer. Nat Rev Cancer 1: 55-67

Funk JO (1999) Cancer cell cycle control. Anticancer Res 19: $4772-4780$

Galizia G, Lieto E, De Vita F, Romano C, Orditura M, Castellano P, Imperatore V, Infusino S, Catalano G, Pignatelli C (2002) Circulating levels of interleukin-10 and interleukin- 6 in gastric and colon cancer patients before and after surgery: relationship with radicality and outcome. J Interferon Cytokine Res 22: 473-482

Garrido F, Algarra I (2001) MHC antigens and tumor escape from immune surveillance. Adv Cancer Res 83: 117-158

Heriot AG, Marriott JB, Cookson S, Kumar D, Dalgleish AG (2000) Reduction in cytokine production in colorectal cancer patients: association with stage and reversal by resection. $\mathrm{Br} J$ Cancer 82: $1009-1012$

Higaki S, Akazawa A, Nakamura H, Yanai H, Yoshida T, Okita K (1999) Metaplastic polyp of the colon develops in response to inflammation. J Gastroenterol Hepatol 14: 709-714

Itzkowitz SH, Yio X (2004) Inflammation and cancer IV. Colorectal cancer in inflammatory bowel disease: the role of inflammation. Am J Physiol Gastrointest Liver Physiol 287: G7-17
Jacobson-Brown P, Neuman MG (2004) Colorectal polyposis and immunebased therapies. Can J Gastroenterol 18: 239-249

Kidd P (2003) Th1/Th2 balance: the hypothesis, its limitations, and implications for health and disease. Altern Med Rev 8: 223-246

Koornstra JJ, de Jong S, Hollema H, de Vries EG, Kleibeuker JH (2003) Changes in apoptosis during the development of colorectal cancer: a systematic review of the literature. Crit Rev Oncol Hematol 45: $37-53$

Koornstra JJ, Rijcken FE, de Jong S, Hollema H, de Vries EG, Kleibeuker JH (2004) Assessment of apoptosis by M30 immunoreactivity and the correlation with morphological criteria in normal colorectal mucosa adenomas and carcinomas. Histopathology 44: 9-17

Leslie A, Carey FA, Pratt NR, Steele RJ (2002) The colorectal adenomacarcinoma sequence. $\mathrm{Br}$ J Surg 89: $845-860$

Macarthur M, Hold GL, El Omar EM (2004) Inflammation and Cancer II. Role of chronic inflammation and cytokine gene polymorphisms in the pathogenesis of gastrointestinal malignancy. Am J Physiol Gastrointest Liver Physiol 286: G515-G520

Matsuda M, Salazar F, Petersson M, Masucci G, Hansson J, Pisa P Zhang QJ, Masucci MG, Kiessling R (1994) Interleukin 10 pretreatment protects target cells from tumor- and allo-specific cytotoxic $\mathrm{T}$ cells and downregulates HLA class I expression. J Exp Med 180: $2371-2376$

Nagorsen D, Scheibenbogen C, Letsch A, Germer CT, Buhr HJ, HegewischBecker S, Rivoltini L, Thiel E, Keilholz U (2005) T cell responses against tumor associated antigens and prognosis in colorectal cancer patients. J Transl Med 3: 3

Naito Y, Saito K, Shiiba K, Ohuchi A, Saigenji K, Nagura H, Ohtani H (1998) CD8+ T cells infiltrated within cancer cell nests as a prognostic factor in human colorectal cancer. Cancer Res 58: $3491-3494$

Nakamori M, Iwahashi M, Nakamura M, Ueda K, Zhang X, Yamaue $\mathrm{H}$ (2003) Intensification of antitumor effect by $\mathrm{T}$ helper 1-dominant adoptive immunogene therapy for advanced orthotopic colon cancer. Clin Cancer Res 9: 2357-2365

Nakayama H, Kitayama J, Muto T, Nagawa H (2000) Characterization of intracellular cytokine profile of $\mathrm{CD} 4(+) \mathrm{T}$ cells in peripheral blood and tumor-draining lymph nodes of patients with gastrointestinal cancer. Jpn J Clin Oncol 30: $301-305$ 
O’Byrne KJ, Dalgleish AG (2001) Chronic immune activation and inflammation as the cause of malignancy. $\mathrm{Br} J$ Cancer 85: $473-483$

O'Hara RJ, Greenman J, MacDonald AW, Gaskell KM, Topping KP, Duthie GS, Kerin MJ, Lee PW, Monson JR (1998) Advanced colorectal cancer is associated with impaired interleukin 12 and enhanced interleukin 10 production. Clin Cancer Res 4: $1943-1948$

Robinson SC, Coussens LM (2005) Soluble mediators of inflammation during tumor development. Adv Cancer Res 93: 159-187

Salminen E, Palmu S, Vahlberg T, Roberts PJ, Soderstrom KO (2005) Increased proliferation activity measured by immunoreactive $\mathrm{Ki67}$ is associated with survival improvement in rectal/recto sigmoid cancer. World J Gastroenterol 11: 3245-3249

Shibata M, Nezu T, Kanou H, Abe H, Takekawa M, Fukuzawa M (2002) Decreased production of interleukin-12 and type 2 immune responses are marked in cachectic patients with colorectal and gastric cancer. J Clin Gastroenterol 34: $416-420$
Sveinbjornsson B, Rushfeldt C, Olsen R, Smedsrod B, Seljelid R (1997) Cytotoxic effect of cytokines on murine colon carcinoma cells involves TNF-mediated apoptosis. Biochem Biophys Res Commun 233: 270-275

Terabe M, Park JM, Berzofsky JA (2004) Role of IL-13 in regulation of antitumor immunity and tumor growth. Cancer Immunol Immunother 53: $79-85$

Tomita K, Okabe S (2005) Exogenous histamine stimulates colorectal cancer implant growth via immunosuppression in mice. J Pharmacol Sci 97: $116-123$

Valera V, Yokoyama N, Walter B, Okamoto H, Suda T, Hatakeyama K (2005) Clinical significance of Ki-67 proliferation index in disease progression and prognosis of patients with resected colorectal carcinoma. Br J Surg 92: $1002-1007$

Weitz J, Koch M, Debus J, Hohler T, Galle PR, Buchler MW (2005) Colorectal cancer. Lancet 365: $153-165$

Zou W (2005) Immunosuppressive networks in the tumour environment and their therapeutic relevance. Nat Rev Cancer 5: 263-274 